\title{
NÍVEL DE ATIVIDADE FÍSICA E RISCO DE QUEDAS EM IDOSOS DA COMUNIDADE
}

\author{
PHYSICAL ACTIVITY LEVEL AND FALL RISK \\ IN THE COMMUNITY ELDERLY
}

\begin{abstract}
Dinara Hansen', Tatiane Konrad Rückert², Carolina Böettge Rosa ${ }^{3}$, Solange Beatriz Billig Garces ${ }^{4}$, Michele Ferraz Figueiró ${ }^{5}$, Jociane de Carvalho Myskiw ${ }^{6}$, Janaina Coser ${ }^{7}$, Ângela Vieira Brunelli
\end{abstract}

\section{RESUMO}

A proposta deste estudo foi verificar a associação entre o risco de quedas de 127 idosos da comunidade e o nível de atividade física. O risco de quedas foi avaliado através dos instrumentos Fall Risk Score de Downton, teste Timed Up and Go (TUG) e o Teste de Alcance Funcional (TAF) e o nível de atividade física utilizando o Questionário Internacional de Atividade Física (IPAQ) versão curta. A maioria dos idosos entrevistados $(88,7 \%)$ foram classificados como ativos ou muito ativos e sem risco de quedas (TUG 89,8\%, TAF 97,6\%, Downton 64,5\%). A relação entre o nível de atividade física e risco de quedas através dos testes TUG e Downton mostrou-se significativa, estando os idosos mais ativos com menor risco de quedas e todos os instrumentos que avaliaram este risco apresentaram associação significativa entre si demonstrando que a atividade física pode reduzir o risco de quedas, minimizando perdas funcionais do envelhecimento.

Descritores: Envelhecimento; Fatores de Risco; Acidentes por Quedas; Estilo de Vida.

\begin{abstract}
The purpose this study was verify the association between the risk of falls of 127 elderly of community and the level of physical activity. The risk of falls was assessment through the instruments Fall Risk Score of Downton, Timed Up and Go Test (TUG) and Functional Reach Test (FRT) and the level of physical activity using the short version International Physical Activity Questionnaire (IPAQ). The majority of elderly respondents (88.7\%) was classified as active or very active and without risk of falls (TUG 89.8\%, FRT 97.6\% and Downton 64.5\%). The relation between the level of physical activity and the risk of falls through the TUG and Downton has been shown significant, being the most active elderly with less risk of falls and all the instruments that assessed the risks have presented significant association each other demonstrating that physical activity can reduce the risk of falls and minimize functional losses of aging.
\end{abstract}

Descriptors: Aging; Risk Factors; Accidental Falls; Life Style.
${ }^{1}$ Mestre em Gerontologia Biomédica pela Pontifícia Universidade Católica do Rio Grande do Sul (PUCRS), Porto Alegre, RS, Brasil.

\section{${ }^{2}$ Bacharel em Fisioterapia pela Universidade de Cruz Alta} (UNICRUZ), Cruz Alta, RS, Brasil.

\section{${ }^{3}$ Mestre em Patologia pela} Universidade Federal de Ciências da Saúde de Porto Alegre (UFCSPA), Porto Alegre, RS, Brasil.

${ }^{4}$ Doutora em Ciências Sociais pela Universidade do Vale do Rio dos Sinos (UNISINOS), São Leopoldo, RS, Brasil.

${ }^{5}$ Pós Doutora em Estatística Aplicada pela Universidade de São Paulo (USP), São Paulo, SP, Brasil.

${ }^{6}$ Doutora em Medicina e Ciências da Saúde pela Pontifícia Universidade Católica do Rio Grande do Sul (PUCRS), Porto Alegre, RS, Brasil.

${ }^{7}$ Doutora em Biologia Celular e Molecular Aplicada à Saúde pela Universidade Luterana do Brasil (ULBRA), Canoas, RS, Brasil.

${ }^{8}$ Mestre em Extensão Rural pela Universidade Federal de Santa Maria (UFSM), Santa Maria, RS, Brasil. 


\section{Introdução}

O aumento da população de idosos no Brasil é acompanhado de um aumento dos índices de mortalidade por quedas ${ }^{1}$. Aproximadamente $30 \%$ dos indivíduos com mais de 65 anos de idade cai ao menos uma vez por ano e metade de forma recorrente. Estas quedas, para os idosos, possuem um significado muito relevante, pois podem levá-lo à incapacidade, injúria e morte?2.

Além do aumento do número de pessoas com 60 anos ou mais, a população está se tornando cada vez mais longeva, pois segundo pesquisas do Instituto Brasileiro de Geografia e Estatística (IBGE), a expectativa de vida no País, que alcançava 41,5 anos sete décadas atrás, atualmente ultrapassa os 73 anos $^{3}$.

Assim, estudar a problemática associada às quedas em pessoas idosas constitui uma temática relevante e desafiadora, que contribui para a promoção do bem-estar dos idosos tanto no Brasil, quanto na maioria das nações desenvolvidas, por ser 0 envelhecimento uma preocupação coletiva ${ }^{4,5}$.

A queda é definida como um evento não intencional, que tem como resultado a mudança da posição inicial do indivíduo para um mesmo nível ou nível mais baix $0^{6}$. Este evento pode resultar em consequências físicas ${ }^{2}$, funcionais ${ }^{7}$ e psicossociais ${ }^{8}$, contribuindo para o declínio funcional, decorrente das lesões ósseas e musculares associadas, da limitação da atividade física diária e do receio que o indivíduo tem de sofrer novas quedas, levando-o as limitações de mobilidade e dependência para atividades da vida diária (AVDs).

Percebe-se então a necessidade da identificação do risco de quedas e fatores associados em idosos possibilitando planejamento de estratégias preventivas, de reorganização ambiental e de reabilitação funcional ${ }^{4}$, tornando-se prioritária a abordagem multiprofissional e interdisciplinar, a fim de minimizar o risco de quedas, evitar a dependência e diminuir a morbimortalidade dos idosos 9 .

Fatores de risco apontados como determinantes de quedas são: idade igual ou maior a 75 anos, sexo feminino, presença de declínio cognitivo, de inatividade, de sarcopenia e de distúrbios do equilíbrio corporal, marcha ou de mobilidade, história prévia de acidente vascular encefálico (AVE), de quedas anteriores e de fraturas, comprometimento na capacidade de realizar atividades de vida diária e o uso de medicações psicotrópicas, em especial os benzodiazepínicos, assim como o uso de várias medicações concomitantes ${ }^{10}$.

Destacam-se os fatores que estão associados às perdas físicas e funcionais naturais do processo de envelhecimento, as quais podem ser agravadas pela inatividade física. Neste sentido, a atividade física surge como um aliado na manutenção da capacidade funcional dos idosos, distanciamento das características que levam a fragilidade e provocando aumento da função imunológica do organismo ${ }^{11}$. Segundo o estudo de Netto e Guimar (2012) ${ }^{12}$, a atividade física possibilita ao idosos o conhecimento e percepção do seu próprio corpo, gerando maior interação social e respostas decorrentes de adaptações fisiológicas e psicológicas, como menor cansaço do corpo ao longo do dia e maior disposição para a realização das tarefas diárias.

Alterações na mobilidade são problemas comuns entre os idosos, pois estas levam à limitação na realização das atividades da vida diária. Neste contexto se insere o profissional fisioterapeuta visando melhorar a funcionalidade e controle da deambulação, suficiente para tornar o idoso seguro e independente. Porém, para que ocorra uma intervenção precisa e eficaz, é necessário conhecer os idosos que são mais vulneráveis e quais os fatores que estão associados às alterações de mobilidade e equilíbrio como a sacopenia, alterações posturais, osteoarticulares e sensoriais e nível de atividade física ${ }^{13}$.

Ante 0 exposto, tendo em vista a grande relevância do tema para a área da fisioterapia, que atua diretamente na prevenção da saúde e reabilitação das fraturas resultantes das quedas, percebeu-se a necessidade de verificar se há relação entre o risco de quedas e nível de atividade física em idosos de duas Estratégias de Saúde da Família (ESF) do município de Cruz Alta - RS para assim, propor estratégias necessárias de prevenção às quedas.

\section{Metodologia}

A presente investigação caracteriza-se como sendo epidemiológica, observacional, analítica e descritiva, com abordagem quantitativa. A população dessa pesquisa foi constituída por aproximadamente 700 idosos cadastrados em duas ESFs de Cruz Alta. Para calcular a amostra foi utilizado cálculo de tamanho de uma amostra aleatória simples e erro amostral de $10 \%$, totalizando 142 idosos. 
Os critérios de exclusão utilizados foram: idosos com doença cardiovascular descontrolada identificada através de autor-relatos de pressão arterial sistêmica descompensada e/ou abandono do tratamento medicamentoso assim como diabetes melittus descompensada; aqueles que não conseguiram assumir as posições nos testes ou utilizaram próteses ou órteses para auxiliar na deambulação; aqueles que não foram encontrados nas suas residências em duas visitas e aqueles que não atingiram o escore mínimo no Mini Exame do Estado Mental (MEEM). O ponto de corte utilizado para analfabetos foi de 15 pontos, para sujeitos com escolaridade entre 1 e 11 anos foi 22 pontos e para sujeitos com escolaridade superior a 11 anos foi 27 pontos $^{7}$.

A seleção da amostra foi realizada de forma aleatória, através do contato com os agentes comunitários de saúde das ESFs, que forneceram listas de endereços dos idosos cadastrados, os quais foram visitados em suas residências. Nas visitas, os pesquisadores informaram aos idosos sobre a pesquisa e aqueles que aceitaram participar, assinaram 0 Termo de Consentimento Livre e Esclarecido (TCLE), responderam ao questionário contendo dados sócio demográficos, International Physical Activity Questionnaire - IPAQ, Escala de Risco de Quedas de Downton e realizaram os testes Timed Up And Go (TUG) e Teste de Alcance Funcional (TAF) na sua própria residência. Para os idosos analfabetos, o TCLE foi lido pelo pesquisador, sendo esclarecidas todas as dúvidas, para posterior impressão da digital do polegar ou, na presença de familiar, era solicitada assinatura a rogo como testemunha.

Num primeiro momento os idosos realizaram o Mini Exame do Estado Mental utilizado como teste de rastreio para perda cognitiva. Para avaliar o nível de atividade física foi utilizado o IPAQ versão curta, validado no Brasil, apresentando níveis adequados de reprodutibilidade, validade aceitável e maior praticidade ${ }^{14}$. Os indivíduos avaliados foram classificados em sedentários, insuficientemente ativos, ativo e muito ativo, conforme a duração e a frequência das atividades leves, moderadas ou vigorosas realizadas na última semana ${ }^{7}$.

Para avaliar o risco de quedas foi utilizada a escala de Downton, já validado em português, enfocando quedas anteriores, administração de medicamentos, déficit sensorial, estado mental e deambulação. Idosos com escores maiores de 3 pontos, num total de 11 , indicaram risco de quedas ${ }^{14}$.

Os testes TUG e TAF foram utilizados para avaliar, nos idosos, o risco de quedas e o equilíbrio corporal dinâmico. No TUG o tempo gasto pelos idosos para levantar de uma cadeira sem braço, percorrer uma distância de três metros, retornar e sentar na mesma cadeira foi cronometrado. Idosos que realizaram o teste em até 10 segundos (s) não apresentaram risco de quedas; idosos que levaram entre 11 a 20 s demonstraram baixo risco de quedas e tempos maiores que 20s indicaram que os idosos apresentavam alto risco de quedas ${ }^{14}$.

No TAF, o idoso descalço, com os pés no chão, colocou-se lateralmente a uma parede na qual se encontrava uma fita métrica, fixada na posição horizontal. $O$ idoso posicionou o membro superior em flexão de ombro a $90^{\circ}$, com centro da articulação do ombro coincidindo com o zero centímetro da fita. Foi medido o tamanho do braço do idoso com a mão aberta e os dedos esticados até a ponta do dedo médio. Partindo dessa posição, foi feita inclinação máxima do tronco à frente com o membro superior alcançando a maior distância possível, sem apoiar na parede e/ou na fita métrica, sem apoio e sem retirar o calcanhar do chão. A distância percorrida, em centímetros, entre a medida inicial do tamanho do braço e a final do deslocamento do tronco até a ponta do dedo médio foi o alcance funcional do idoso. Utilizou-se a maior distância alcançada dentre três tentativas, sendo considerado idoso com risco de quedas aquele com alcance menor do que 15 centímetros (cm).

A análise dos dados foi realizada no programa estatístico Statistical Package for the Social Sciences (SPSS) versão 22. Para a análise univariada das variáveis qualitativas, foram elaboradas tabelas de distribuição de frequências, enquanto que para a análise bivariada, foram construídas tabelas de contingência. A medida da associação entre as variáveis qualitativas foi estimada através do teste Qui-Quadrado de Pearson e do teste exato de Fisher (para valores esperados menores que 5 e tabela $2 \times 2$ ).

Na comparação múltipla entre as médias de idades dos três grupos de risco de queda do instrumento TUG, foi utilizada a análise de variância (ANOVA) com teste post hoc de Tukey. Utilizou-se o teste te Student para amostras independentes, na comparação entre as médias de idades dos dois grupos de risco de quedas dos instrumentos Downton e TAF. Considerou-se estatisticamente significativo um valor de $p<0,05$.

A pesquisa foi conduzida segundo a resolução específica do Conselho Nacional de Saúde (466/2012) e o projeto foi aprovado pelo Comitê de Ética em Pesquisa da UNICRUZ pelo parecer número 1.026.858 e C.A.A.E. 33015814.0.0000.5322. 


\section{Resultados}

Neste estudo foram analisados os dados referentes a 142 pessoas com mais de 60 anos do município de Cruz Alta, cadastradas em duas ESF do município. No entanto, entre os idosos com $1^{\circ}$ grau incompleto e ensino médio completo, $8,9 \%$ ( $n=11$ ) tiveram algum comprometimento cognitivo e entre os idosos com ensino superior incompleto ou maior escolaridade, $25 \%(n=4)$ tiveram comprometimento cognitivo, os quais foram excluídos da amostra pelo fato de não terem atingido os escores mínimos no MEEM.

Assim, a amostra final foi composta de 127 idosos, não havendo mais nenhuma necessidade de exclusões por outros critérios. Deste total, $70,9 \%(n=90)$ eram do sexo feminino. A média de idade destes idosos foi de 70,44 anos com desvio padrão de 7,28 anos, cujas idades mínima e máxima foram de 60 anos e 93 anos, respectivamente. A faixa etária predominante foi de 60 a 79 anos para $55,9 \%(n=71)$ dos idosos.

Entre os 127 idosos, predominaram nível de escolaridade ensino fundamental incompleto com $60,6 \%$ ( $n=77$ ) dos idosos, a renda mensal de um salário mínimo para $64,6 \%(n=82)$ dos idosos e estado civil casado para $48 \%$ ( $n=61)$ dos idosos. A caracterização completa da amostra pode ser visualizada na Tabela 1.

Tabela 1 - Caracterização da amostra de idosos de duas ESFs de Cruz Alta-RS, 2014

\begin{tabular}{|c|c|c|}
\hline Variável & $\mathbf{N}$ & $\%$ \\
\hline \multicolumn{3}{|l|}{ Sexo } \\
\hline Feminino & 90 & 70,9 \\
\hline Masculino & 37 & 29,1 \\
\hline Total & 127 & 100 \\
\hline \multicolumn{3}{|l|}{ Idade (anos) } \\
\hline 60 a 69 & 71 & 55,9 \\
\hline 70 a 79 & 38 & 29,9 \\
\hline 80 a 89 & 17 & 13,4 \\
\hline+90 & 01 & 0,8 \\
\hline Total & 127 & 100 \\
\hline \multicolumn{3}{|l|}{ Escolaridade } \\
\hline Analfabeto & 03 & 2,4 \\
\hline Ensino Fundamental Incompleto & 77 & 60,6 \\
\hline Ensino Fundamental completo & 19 & 15,0 \\
\hline Ensino Médio Completo & 19 & 15,0 \\
\hline Ensino Superior Completo & 09 & 7,0 \\
\hline Total & 127 & 100 \\
\hline \multicolumn{3}{|l|}{ Estado civil } \\
\hline Casado & 61 & 48,0 \\
\hline Solteiro & 14 & 11,0 \\
\hline Viúvo & 43 & 33,9 \\
\hline Separado & 09 & 7,1 \\
\hline Total & 127 & 100 \\
\hline
\end{tabular}




\begin{tabular}{|c|c|c|}
\hline Variável & $\mathbf{N}$ & $\%$ \\
\hline \multicolumn{3}{|c|}{ Renda (salário mínimo) } \\
\hline Sem renda & 04 & 3,1 \\
\hline 1 & 82 & 64,6 \\
\hline 2 & 19 & 15,0 \\
\hline 3 a 5 & 11 & 8,7 \\
\hline+5 & 11 & 8,7 \\
\hline Total & 127 & 100 \\
\hline \multicolumn{3}{|c|}{ Situação atual de trabalho } \\
\hline Trabalha & 08 & 6,3 \\
\hline Aposentado & 73 & 57,5 \\
\hline Pensionista & 25 & 19,7 \\
\hline Outra & 21 & 16,5 \\
\hline Total & 127 & 100 \\
\hline
\end{tabular}

Os dados referentes à avaliação do risco de quedas realizada através dos instrumentos TUG, TAF e Downton, podem ser visualizados na tabela 2. Verificou-se, através do teste TUG, que 60,6\% $(n=84)$ dos idosos apresentaram algum grau de risco quando somados risco baixo e alto de quedas enquanto que através dos testes TAF e Downton, $97,6 \%(n=124)$ e $64,5 \%$ ( $n=82)$ dos idosos, respectivamente, não apresentaram risco de quedas. Cabe destacar que a média do tempo gasto para a realização do TUG foi de $13,07( \pm 4,89)$ segundos, que classifica os idosos com baixo risco de quedas e a média do TAF foi de $28,72( \pm 7,54) \mathrm{cm}$.

Tabela 2 - Riscos de quedas dos idosos de duas ESFs de Cruz Alta-RS, 2014

\begin{tabular}{c|c|c}
\hline \multicolumn{3}{|c}{ N } \\
\hline \multicolumn{2}{|c|}{ Timed Up and Go } \\
\hline Sem risco & 43 & 33,9 \\
\hline Baixo Risco & 71 & 55,9 \\
\hline Alto Risco & 13 & 10,2 \\
\hline Total & 127 & 100 \\
\hline Teste do Alcance Funcional & & \\
\hline Sem risco & 124 & 97,6 \\
\hline Com Risco & 03 & 2,4 \\
\hline Total & 127 & 100 \\
\hline Downton & 82 & 64,5 \\
\hline Sem Risco & 45 & 35,5 \\
\hline Com risco & 127 & 100 \\
\hline Total & &
\end{tabular}


Segundo o teste Qui-Quadrado de Pearson, $a=5 \%$, evidenciou-se uma relação de dependência estatisticamente significativa entre as variáveis qualitativas instrumento Downton e sexo $(p=0,037)$. No entanto, não se verificou nenhuma relação de dependência estatisticamente significativa entre as variáveis sexo e instrumento TUG $(p=0,776)$. De acordo com o teste exato de Fisher, $\alpha=5 \%$, não se evidenciou nenhuma relação de dependência estatisticamente significativa entre as variáveis qualitativas instrumento sexo e instrumento TAF $(p=0,556)$.

Na tabela 3, estão as medidas descritivas da variável idade para os instrumentos Downton, TAF e TUG. De acordo com o teste $t$ de Student para amostras independentes, $a=5 \%$, não houve nenhuma diferença estatisticamente significativa entre as médias das idades dos grupos Sem Risco e Com Risco do instrumento Downton ( $p=0,701)$, assim como, entre as médias de idade dos grupos Sem Risco e Com Risco do instrumento TAF ( $p=0,351)$.

$\mathrm{Na}$ tabela 3 , de acordo com teste $\mathrm{F}$ da ANOVA, $a=5 \%$, há pelo menos uma diferença estatisticamente significativa entre as médias de idade dos grupos instrumento TUG $(p=0,000)$. O teste de Tukey indicou que o grupo Alto Risco de quedas apresentou maior média para a variável idade quando comparada aos grupos Baixo Risco $(p=0,000)$ e Sem Risco $(p=0,000)$.

Tabela 3 - Medidas descritivas de idade para os instrumentos de avaliação do risco de quedas de idosos de duas ESFs Cruz Alta-RS, 2014

\begin{tabular}{|c|c|c|c|c|c|}
\hline \multicolumn{6}{|c|}{ Medidas descritivas de idade (anos) } \\
\hline Risco de quedas & Mínima & Máxima & Média & DP & Valor P \\
\hline \multicolumn{6}{|l|}{ Downton } \\
\hline Sem Risco & 60 & 93 & 70,26 & 7,09 & \multirow{2}{*}{0,701} \\
\hline Com risco & 60 & 88 & 70,78 & 7,68 & \\
\hline \multicolumn{6}{|l|}{ TAF } \\
\hline Sem risco & 60 & 93 & 70,35 & 7,17 & \multirow{2}{*}{0,351} \\
\hline Com Risco & 64 & 88 & 74,33 & 12,34 & \\
\hline \multicolumn{6}{|l|}{ TUG } \\
\hline Sem risco & 60 & 78 & 67,47 & 4,49 & \multirow{3}{*}{0,000} \\
\hline Médio risco & 60 & 80 & 70,34 & 6,78 & \\
\hline Alto Risco & 65 & 93 & 80,85 & 8,31 & \\
\hline
\end{tabular}

Ao avaliar o nível de atividade física, constatou-se que $33 \%(n=42)$ dos idosos foram classificados como muito ativos, $43,3 \%$ ( $n=55)$ como ativos, 18,9\% ( $n=24)$ irregularmente ativos e somente 4,7\% $(n=6)$ dos idosos como sedentários.

O teste Qui-Quadrado de Pearson, $a=5 \%$, indicou uma relação de dependência estatisticamente significativa entre o nível de atividade física e os instrumentos Downton ( $p=0,032)$ e TUG $(p=0,000)$. No entanto, entre o nível de atividade física e o TAF, não se evidenciou nenhuma relação de dependência $(p=0,072)$. Estes resultados podem ser visualizados na tabela 4 .

Tabela 4 - Relação entre Atividade Física e Risco de Quedas de idosos de duas ESFs de Cruz Alta-RS, 2014

\begin{tabular}{|c|c|c|c|c|c|c|}
\hline \multicolumn{7}{|c|}{ Nível de Atividade Física } \\
\hline $\begin{array}{l}\text { Risco de } \\
\text { quedas }\end{array}$ & $\begin{array}{l}\text { Muito } \\
\text { Ativo } \\
\text { n (\%) }\end{array}$ & $\begin{array}{l}\text { Ativo } \\
\text { n (\%) }\end{array}$ & $\begin{array}{c}\text { Irregularmente } \\
\text { ativo } \\
\text { n (\%) }\end{array}$ & $\begin{array}{c}\text { Sedentário } \\
\text { n (\%) }\end{array}$ & $\begin{array}{l}\text { Total } \\
\text { n (\%) }\end{array}$ & $\begin{array}{c}\text { Valor } \\
\mathbf{p}\end{array}$ \\
\hline \multicolumn{7}{|l|}{ Downton } \\
\hline Sem Risco & $40(31,5)$ & $28(22,0)$ & $13(10,2)$ & $1(0,8)$ & $82(64,5)$ & \multirow{3}{*}{0,032} \\
\hline Com risco & $15(11,8)$ & $14(11,0)$ & $11(8,7)$ & $5(3,90)$ & $45(35,5)$ & \\
\hline Total & $55(43,3)$ & $42(33,0)$ & $24(18,9)$ & $6(4,70)$ & $127(100)$ & \\
\hline
\end{tabular}

continua... 


\begin{tabular}{|c|c|c|c|c|c|c|}
\hline \multicolumn{7}{|c|}{ Nível de Atividade Física } \\
\hline $\begin{array}{l}\text { Risco de } \\
\text { quedas }\end{array}$ & $\begin{array}{l}\text { Muito } \\
\text { Ativo } \\
\text { n (\%) }\end{array}$ & $\begin{array}{l}\text { Ativo } \\
\text { n (\%) }\end{array}$ & $\begin{array}{c}\text { Irregularmente } \\
\text { ativo } \\
\text { n }(\%)\end{array}$ & $\begin{array}{c}\text { Sedentário } \\
\text { n (\%) }\end{array}$ & $\begin{array}{l}\text { Total } \\
\text { n (\%) }\end{array}$ & $\begin{array}{c}\text { Valor } \\
\text { p }\end{array}$ \\
\hline \multicolumn{7}{|l|}{ TAF } \\
\hline Sem Risco & $55(43,3)$ & $41(32,3)$ & $23(18,1)$ & $05(3,90)$ & $124(97,6)$ & \multirow{3}{*}{0,072} \\
\hline Com risco & $0(0,00)$ & $01(0,8)$ & $01(0,8)$ & $01(0,8)$ & $03(2,4)$ & \\
\hline Total & $55(43,3)$ & $42(33,1)$ & $24(18,8)$ & $06(4,7)$ & $127(100)$ & \\
\hline \multicolumn{7}{|l|}{ TUG } \\
\hline Sem risco & $23(18,1)$ & $13(10,2)$ & $07(5,50)$ & $0(0,00)$ & $43(33,8)$ & \multirow{4}{*}{0,000} \\
\hline Médio risco & $31(24,4)$ & $27(21,3)$ & $11(8,70)$ & $02(1,60)$ & $71(56,0)$ & \\
\hline Alto Risco & $01(0,8)$ & $02(1,6)$ & $06(4,70)$ & $04(3,10)$ & $13(10,2)$ & \\
\hline Total & $55(44,3)$ & $42(33,1)$ & $24(18,9)$ & $06(4,70)$ & $127(100)$ & \\
\hline
\end{tabular}

Para complementar o estudo foi verificada a relação entre os instrumentos utilizados para avaliar o risco de quedas dos idosos. O teste Qui-Quadrado de Pearson, $\alpha=5 \%$, indicou uma relação de dependência estatisticamente significativa entre os instrumentos, que avaliaram o risco de quedas, TUG e Downton $(p=0,009)$, assim como, entre os intrumentos TUG e TAF ( $p=0,004)$. Do mesmo modo, segundo o teste exato de Fisher, verificou-se uma relação de dependência estatisticamente significativa entre os instrumentos Downton e TAF $(p=0,043)$.

\section{Discussão}

Em relação aos dados apurados neste estudo é possível perceber predomínio do sexo feminino, faixas etárias de idosos mais jovens, maior número de casados e viúvos bem como baixo nível de escolaridade e aposentados. Dentre estas características, sexo feminino, ser viúvo, ter baixa escolaridade e estar aposentado relacionam-se com a maior predisposição às quedas $^{15}$, pois são considerados fatores limitantes e incapacitantes para a vida do idoso $0^{7,16}$.

O predomínio de mulheres entre os indivíduos investigados vem ao encontro com os dados do IBGE $^{3}$ que apontam para um processo de feminilização da velhice cada vez mais evidente e uma expectativa de vida de 7 anos a mais das mulheres quando comparada aos homens ${ }^{3}$. Outro fator que pode ter contribuído para o predomínio de mulheres neste estudo foi que as mesmas eram encontradas com maior frequência em suas residências e aceitaram, mais facilmente, participar da pesquisa.

Quanto à prática de atividades físicas podemos perceber que o maior percentual de idosos foi classificado como ativo ou muito ativo, o que vai de encontro com os achados de Toscano e Oliveira ${ }^{17}$ onde $65 \%$ das idosas de seu estudo eram ativas. Esta condição favorece o envelhecimento saudável, com redução dos prejuízos que podem ser causados pelo sedentarismo, minimizando assim a ocorrência de quedas.

A prática de atividade física interfere no evento queda em idosos, pois, contribui para a manutenção da aptidão física nestes indivíduos, além de atenuar e reverter a perda de massa muscular ${ }^{12}$. No estudo realizado por Leite, Catistoni e Kirchner ${ }^{18}$ a maioria dos idosos investigados realizava atividades regulares, estando estas, diretamente relacionadas com a manutenção da capacidade funcional. Da mesma forma, no presente estudo também predominaram idosos ativos, o que pode estar relacionado com o predomínio de idosos mais jovens, que na maioria dos casos, apresentam-se com perdas físico-funcionas que não comprometem a realização atividades cotidianas e favorecem o envelhecimento mais ativo e saudável.

Segundo Delbare et $\mathrm{a}$, a prática de atividades regulares é mais comum entre idosos mais jovens, com menos de 70 anos de idade, do que entre idosos mais velhos. Almeida et al ${ }^{9}$, em seu estudo também obtiveram resultados que vão de encontro com estes dados, onde cerca de $40 \%$ dos idosos mais velhos indicaram que seu estado de saúde prejudicava a prática de atividade física e portanto deixavam de realizá-la. Estes achados se solidificam através dos 
achados de Thibaud, et a ${ }^{19}$, os quais demostram que, à medida que aumenta a idade cronológica dos idosos, estes tornam se menos ativos e suas capacidades físicas diminuem.

$\mathrm{O}$ fato de predominarem na amostra, idosos de faixas etárias mais jovens e que se mantem ativos pode justificar os achados referentes ao baixo número de idosos com risco aumentado para quedas, identificado através dos três diferentes instrumentos de avaliação utilizados. Contrário a estes achados, o estudo realizado por Reis, Nunes e Flores ${ }^{20}$, que também aplicou a escala de risco de quedas de Downton, apresentou elevado percentual de idosos com risco alto para queda (94\%), mesmo com média de idade baixa (69 anos), porém, não levou em consideração o nível de atividade física e desempenho físico destes indivíduos.

A maior prevalência de idosos que não apresentaram risco aumentado para quedas, quando avaliados através do TUG, concorda com os resultados de Karuka et.al. ${ }^{21}$ e Santos, Borges e Menezes ${ }^{22}$, que encontraram médias de 13 ( \pm $4,89)$ segundos e $7,61( \pm 1,56)$ segundos, respectivamente, para a realização do teste, as quais também demonstraram que a maioria dos idosos não apresentava risco aumentado de quedas.

Quando avaliado o desempenho com os resultados de TAF, os idosos do estudo de Fidelis, Patrizzi \& Walsh ${ }^{23}$ apresentaram médias de 33,46 $( \pm 8,4)$ centímetros sendo bem maiores que os valores referidos na literatura de 15 centímetros, que indicam maior propensão às quedas, vindo de encontro com os resultados do nosso estudo onde a média foi de $28,72( \pm 7,54)$ centímetros.

Os achados referentes à independência entre médias de idade e resultado do instrumento de Downton e desempenho no TAF não foram encontrados em outros estudos. Já, achados semelhantes da relação significativa entre idade e desempenho no TUG foram verificados em estudo de Santos ${ }^{21}$ no qual os grupos etários investigados apresentaram diferenças significativas, mostrando que quanto maior a idade, maior o déficit de equilíbrio e, consequentemente, maior o risco de quedas.

Maciel e Guerra ${ }^{24}$ em seu estudo também observaram forte associação da alteração da mobilidade pelo TUG, com a variável idade, onde a maioria dos idosos pertencentes às faixas etárias acima dos 75 anos ou mais teve maior chance de apresentar déficit na mobilidade, com consequente risco de quedas aumentado.

Quando relacionamos a variável sexo com os instrumentos Downton, TUG e TAF verificou-se não haver nenhuma relação significativa, porém na literatura encontramos que o risco de uma mulher sofrer queda é de 1,71 vezes maior quando comparada ao homem, pois as mulheres estão mais expostas a atividades externas, utilização acentuada de fármacos, uso de psicotrópicos e diminuição da força de preensão 4 .

Podemos destacar que em nosso estudo predominaram idosos muito ativos e ativos sem risco aumentado de quedas o que vai de encontro com os achados de Pinho, et al. ${ }^{4}$ que também apresentou uma relação significativa entre o nível de atividade física e o risco de quedas.

Não podemos afirmar que a idade avançada e as perdas funcionais sejam causas únicas para quedas. Existe sim uma combinação de fatores intrínsecos e extrínsecos, sendo que uma parte dessas quedas ocorre por inadequações no ambiente 4 . Assim, a atividade física se torna uma aliada à interação social, melhorando a autoeficácia e proporcionando uma maior sensação de controle sobre os eventos e as demandas do meio, diminuindo, assim, os ricos de quedas entre os idosos da comunidade ${ }^{15}$.

A relação verificada entre as variáveis nível de atividade física e desempenho no TUG pode ser justificada pelo fato de o TUG estar relacionado com equilíbrio, velocidade de marcha e capacidade funcional, os quais sofrem influência direta da realização de atividade física. Assim, os idosos que se mantem ativos tendem a apresentar melhor desempenho no TUG e automaticamente menor propensão às quedas. Estes, normalmente têm idades menos avançadas, pois são os mesmos que apresentam pequenas perdas fisiológicas, que não comprometem diretamente a mobilidade e a realização de atividades cotidianas.

O estudo dos autores Silva, Duarte \& Arantes $^{25}$ também demonstrou haver relação significativa entre o nível de atividade física e TUG, sendo que os idosos mais ativos apresentaram melhores desempenhos no TUG.

A independência entre as variáveis nível de atividade física e desempenho TAF também foi encontrada por Campos, Vianna \& Campos ${ }^{26}$, os quais apontaram como possível justificativa, o fato de os idosos com alterações mais importantes do equilíbrio cercarem-se de maiores cuidados durante a realização do teste, a fim de evitar desequilíbrios e consequentes quedas.

As alterações de equilíbrio podem ser prevenidas através do envelhecimento ativo. Manter-se ativo após os 60 anos de idade impede o declínio gradual das aptidões físicas, que podem alterar os hábitos de vida e rotinas diárias dos idosos, levando-os a realizar atividades e formas de ocupação pouco ativas ${ }^{22}$.

A perda de massa muscular e a diminuição da força dos músculos, fisiológicas do processo de envelhecimento, interferem na autonomia, bem-estar e qualidade de vida dos idosos, as quais ficam mais evidentes entre os idosos com idades entre 70 e 80 anos (perda de $30 \%$ da massa muscular) ${ }^{27}$. Estes achados podem justificar as variações no desempenho dos idosos de diferentes idades ao realizar o TUG, ficando os mais jovens com os melhores resultados. 
A relação de dependência encontrada entre os instrumentos TAF e TUG, que avaliaram o risco de quedas nos idosos, pode estar relacionada ao fato de serem instrumentos que levam em consideração mobilidade funcional, equilíbrio estático e dinâmico. Já a relação de dependência entre estes testes e a escala de Downton pode estar associada ao fato de ser esta uma escala que investiga presença de fatores que podem influenciar no controle do equilíbrio corporal como uso de medicamentos, quedas anteriores, déficit sensorial, estado mental e marcha com ou sem utilização de apoio.

Assim, de acordo com os resultados obtidos em nosso estudo foi possível identificar que a prática de atividade física é capaz de reduzir o risco de quedas, sendo uma das formas alternativas para minimizar as perdas de força muscular com o avanço da idade. A sua prática possibilita ganhos, minimizando e retardando os efeitos do envelhecimento com presença de doenças crônicas, e consequentemente uma melhora e/ou manutenção da autonomia do idoso, sua independência e inserção social22.

Cabe destacar que, como predominaram neste estudo idosos jovens, com ausência de riscos de quedas, percebese a necessidade de repensar estratégias voltadas para a manutenção da funcionalidade e independência destes, para que os mesmos não venham a apesentar significativas alterações físico-funcionais que comprometam sua mobilidade em idades mais avançadas.

\section{Considerações Finais}

Os resultados deste estudo sugerem associação significativa entre o nível de atividade física e o risco de quedas em idosos. Verificou-se que os idosos ativos e muito ativos foram aqueles que apresentaram menor risco de quedas em diferentes instrumentos de avaliação, podendo concluir que os idosos que se mantêm ativos ao longo do envelhecimento, sofrem influência positiva no que se refere à prevenção de quedas, manutenção da funcionalidade e do equilíbrio corporal.

Desta forma, destaca-se o papel do profissional fisioterapeuta e dos demais profissionais da área da saúde, no planejamento de ações que minimizem o risco de quedas para a população de idosos que cresce e vive cada vez mais, através da proposição de estratégias de estímulo a realização de atividade física regular como forma de reduzir agravos que comprometam a funcionalidade dos idosos.

\section{Referências}

1. Rede Interagencial de Informação para a Saúde - RIPSA. Indicadores básicos para a saúde no Brasil: "conceitos e aplicações". 2. ed. Brasília: Organização Pan-Americana da Saúde, 2008.

2. Maia BC, Viana PS, Arantes PAM; Alecar MA. Consequências das quedas em idosos vivendo na comunidade Rev. Bras. Geriatr. Gerontol, 2011; 14(2):381-393.

3. Instituto Brasileiro de Geografia e Estatística - IBGE. Sinopse do Senso Demográ-fico de 2010. Rio de Janeiro, 2011. 4. Pinho TAM, Silva AO, Tura LFR, Moreira MADSP, Gurgel SN, Smith, AAF, et al. Avaliação do risco de quedas em idosos atendidos em Unidade Básica de Saúde. Rev. Esc. Enferm. USP 2012; 46(2):320-327.

5. Silva MF, Goulart NBA, Lanferini FJ, Dias, MMCP. Relação entre os níveis de atividade física e qualidade de vida de idosos sedentários e fisicamente ativos. Rev. Bras. Geriatr. Gerontol, 2012; 15(4):635-642.

6. Gasparotto LPR, Falsarela GR, Coimbra AMV. As quedas no cenário da velhice: conceitos básicos e atualidades da pesquisa em saúde. Rev. Bras. Geriatr. Gerontol, 2014;17(1):201-209.

7. Delbaere K, Close JCT, Mikolaizak AS, Sachdev PS, Brodaty HE, Lord SR. The Falls Efficacy Scale International (FES-I). A comprehensive longitudinal validation study. Age Ageing. 2010; 39(2):210-216.

8. Malini F, Lopes C, Lourenço R. Medo de quedas em idosos: uma revisão da literatura. Rev HUPE, 2014;13(2):38-44.

9. Almeida ST, Soldera CLC, De Carli GA, Gomes I, Resende T L. Analysis of extrinsic and intrinsic factors that predispose elderly individuals to fall. Rev. Assoc. Med. Bras. [online], 2012;58(4): 427-433.

10. Alves LC, Leite IC, Machado CJ. Fatores associados à incapacidade funcional dos idosos no Brasil: análise multinível. Rev Saúde Pública, 2010; 44(3): 468-78.

11. Sousa, SS, Oliveira PS, Oliveira FS, Holanda MAG, Almeida PC, Machado ALG. Study of sociodemographic factors associated with functional dependence in the elderly. Rev. Enferm, 2013; 2(1):44-48.

12. Netto AFL, Guimarães RF. Atividade Física e incidência de quedas em idosos. Rev. Saúde e Desenvolv, 2012; 1(2):28-43. 
13. Cunha A \& Lourenço R. Quedas em idosos: prevalência e fatores associados. Rev. HUPE, Rio de Janeiro, 2014; 13(2):21-29.

14. Pardni R, Matsudo S, Araújo T, Matsudo V, Andrade E, Braggion A, et al. Questionário internacional de atividade física (IPAQ): Estudo de validade e reprodutibilidade no brasil. Rev Bras Ativ Saude. 2001;10:5-18.

15. Organização Mundial Saúde (OMS) [homepage na internet]. Dis ᄀponível em: www.who.org.br. 2010. [Acesso em maio/2015] 16. Batistoni SST, Namba CS. Idade subjetiva e suas relações com o envelhecimento bem sucedido. Psicol. Estud., 2010;15(4):733-742,

17. Toscano JJO, Oliveira ACC. Qualidade de vida em idosos com distintos níveis de atividade física. Rev Bras Med Esporte, 2009;15(3):169-173.

18. Leite MT, Catistoni $D$ e Kirchner RM. Capacidad funcional y nível cognitivo de adultos mayores residentes em uma comunidade em el sur de Brasil. Enferm Global, 2015;14(1):1-11.

19. Thibaud M, Bloch F, Tournoux-Facon C, Brèque C, Rigaud AS, Dugué B, et al. Impact of physical activity and sedentary behaviour on fall risks in older people: a systematic review and meta-analysis of observational studies. Eur Rev Aging Phys Act, 2012; 9 (1):5-15.

20. Reis LA, Nunes NOS \& Flôres CMR. Risco de Quedas em Idosos: comparação entre a Fall Risk Store de Downton e 0 teste de Timed Up and go Test. Rev. InterScientia, 2013; 1 (3):28-38.

21. Karuka AH, Silva JAM. G; Navega MT. Análise da concordância entre instrumentos de avaliação do equilíbrio corporal em idosos. Rev. bras. fisioter. 2011;15(6):460-466.

22. Santos FPV, Borges LL, Menezes RL. Correlação entre três instrumentos de avaliação para risco de quedas em idosos. Fisioter Mov, 2013;26(4):883-894.

23. Fidelis LT, Patrizzi LJ \& Walsh IAP. Influência da prática de exercícios físicos sobre a flexibilidade, força muscular manual e mobilidade funcional em idosos. Rev. Bras. Geriatr. Gerontol, 2013; 16(1):109-116.

24. Maciel ACC \& Guerra RO. Fatores associados à alteração da mobilidade em idosos residentes na comunidade. Rev Bras Fisioter, 2005; 9(1):17-23.

25. Silva EC, Duarte NB, Arante PMM. Estudo da relação entre o nível de atividade física e o risco de quedas em idosas.

Rev. Fisiot. Pesq, 2011;18(1):23-30.

26. Campos MPS, Viana LG \& Campos AF. Os testes de equilíbrio Alcance Funcional e "Timed Up and Go" e o risco de quedas em idosos. Rev Kairós Gerontol, 2013; 16(4):125-138.

27. Picoli, TS, Figueiredo, LL, Patrizzi, LJ. Sarcopenia e envelhecimento, Fisioter Mov, 2011;24(3):455-462.

\section{Dinara Hansen \\ Endereço para correspondência - Rua: Coronel Martins, $n^{\circ} 1329$, Bairro: Centro, CEP: 98005-210, Cruz Alta, RS, Brasil. \\ E-mail: dhansen@unicruz.edu.br \\ Lattes: http://lattes.cnpq.br/0168148898453119}

Tatiane Konrad Rückert - tatiane-kr@hotmail.com

Carolina Böettge Rosa - carolboettge@gmail.com

Solange Beatriz Billig Garces - sgarces@unicruz.edu.br

Michele Ferraz Figueiró - mfigueiro@unicruz.edu.br

Jociane de Carvalho Myskiw - jociane.myskiw@pucrs.br

Janaina Coser - coser@unicruz.edu.br

Ângela Vieira Brunelli - abrunell@uniccruz.edu.br

\section{Enviado em 08 de janeiro de 2016. Aceito em 20 de junho de 2016.}

